\title{
Gamma rays in $L-B$ coordinates at CORONAS-I altitude
}

\author{
R. Bučík ${ }^{1}$, K. Kudela ${ }^{1}$, S. N. Kuznetsov ${ }^{2}$, and I. N. Myagkova ${ }^{2}$ \\ ${ }^{1}$ Institute of Experimental Physics, SAS, 04353 Košice, Slovakia \\ ${ }^{2}$ Institute of Nuclear Physics, Moscow State University, 119899 Moscow, Russia
}

Received: 31 January 2005 - Revised: 26 May 2005 - Accepted: 28 May 2005 - Published: 15 September 2005

\begin{abstract}
We present here observations of gamma rays in the energy range between 3.0 and $8.3 \mathrm{MeV}$ gathered by the SONG instrument aboard low-altitude polar-orbiting satellite CORONAS-I throughout the period March-June 1994. We concentrate on the emissions related to the trapped particles and organize CORONAS-I measurements in the magnetic $L-B$ coordinate system. The spatial distribution of the average gamma-ray counts reveals that the most intense fluxes were observed under the inner radiation belt, at $L<2$, and that they are exclusively confined into the region of stably trapped particles, where daughter gamma rays could result from the interactions within the spacecraft and instrumental matter. In the outer radiation zone $(L \sim 4)$, the enhanced gamma radiation, also detected outside the stably trapping region, shows pronounced longitudinal variations. The observed eastward increase in the gamma-ray count rate suggests quasi-traped energetic (megavolt) electrons as a source of the gamma rays both in the upper atmosphere and in the satellite matter, most likely, through the bremsstrahlung process in the studied energy domain.
\end{abstract}

Keywords. Magnetospheric physics (Energetic particles, precipitating; Energetic particles, trapped; Magnetosphereionosphere interactions)

\section{Introduction}

The most frequently used coordinate system for particle flux mapping is the invariant $L-B$ coordinate system based on the trapped particle motion in a dipolar magnetic field, introduced by Mcllwain (1961). The points in $L-B$ space present different drift shells where particles are confined. $L$ corresponds to the equatorial radius of a drift shell in a dipole, and $B$ is the mirror point magnetic field value. Thus, the $L-B$ coordinate system maps fluxes perpendicular to the magnetic field line, and $B$ dependence reflects the pitch angle distribution. Roederer (1970) discussed that in symmetric or nearly symmetric magnetic fields (as in the magnetosphere below $L \sim 6$ ), all particles that mirror at a given $L$ shell will stay on

Correspondence to: R. Bučík

(rbucik@upjs.sk) the same $L$ at other longitudes, being a function of the local $B$ value; the omnidirectional flux can be well represented in $L-B$ coordinates, as well.

At the top of the Earth's atmosphere or on space platforms, the regions of intense particle fluxes, gamma rays are produced in a variety of physical processes. One component of the gamma radiation can arise from cosmic rays and their secondaries. The cosmic-ray protons, of an energy of $\sim \mathrm{GeVs}$, may initiate the nucleon-meson-electromagnetic cascades, which result in atmospheric and spacecraft-local gamma-ray emission (Dean et al., 1991). This radiation is characterized by latitudinal dependence (e.g. Efimov et al., 1985 ) and relatively smooth variations on time scales of one solar cycle (e.g. Harris et al., 2003). Gamma rays produced by the solar energetic particle impact on the Earth's atmosphere during the intense shock events have been recently reported by several authors (e.g. Share et al., 2002).

The present paper is concerned with gamma-ray components associated with trapped particles in the Earth's radiation belts. Two classes of bremsstrahlung gamma radiation, one from radiation belt electrons stopping in the satellite, and the other from electrons precipitating towards the atmosphere, were first discussed by Imhof et al. (1974). The most intense fluxes have been measured over the polar regions, especially at times of high geomagnetic activity (Imhof, 1981). Satellite observations of bremsstrahlung emission have been used as a remote sensing technique to map the global distribution of electron energy deposit (e.g. Feldman et al., 1996). The detailed time variations of electron precipitation, however, with limited spatial coverage have been achieved from X-ray bremsstrahlung emission in balloon-borne experiments (e.g. Lorentzen et al., 2000; Millan et al., 2002). The trapped energetic protons $(>10 \mathrm{MeV})$ and their secondary neutrons provide the next significant contribution to the the gamma radiation through the activation of the satellite and instrument materials (Chupp, 1976).

Except for external radiation, gamma rays (prompt and delayed) can be produced within the detector system and compose a so-called internal background. All the instant gamma rays can be eliminated by the veto counter, however, gamma rays from long-lived unstable isotopes, making the active shielding technique ineffective because of the finite 


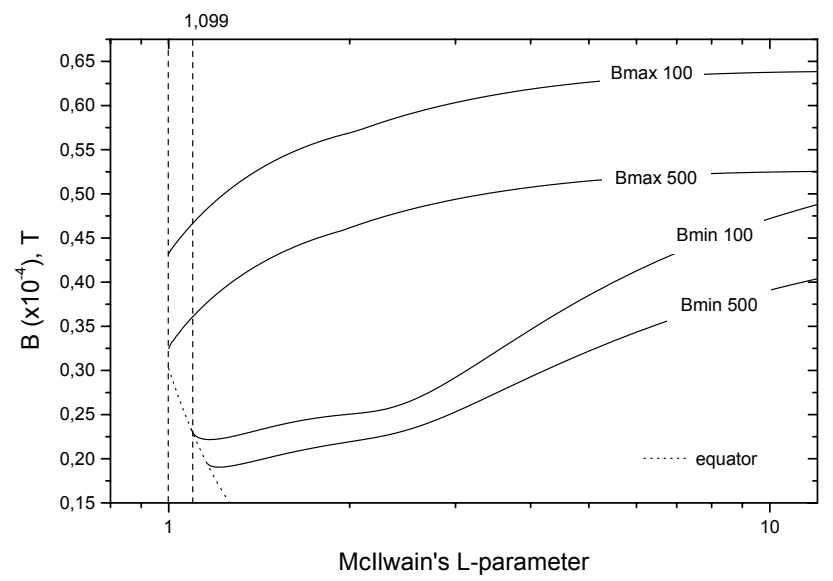

Fig. 1. The levels of 100 and $500 \mathrm{~km}$ altitudes in $L-B$ space. The lines of the minimum and maximum magnetic field strength at 100 (500) km are marked by Bmin 100(500) and Bmax 100(500). The dotted line presents dipole magnetic equator and two dashed vertical lines display $L$ at 1 and 1.099 .

$(<\mu \mathrm{s})$ length of the veto pulse, will be accepted as good events. Measurements by low energy gamma-ray telescopes obtained in SMM and CGRO missions (Share et al., 1989; Johnson et al., 1993), and predictions of Dyer et al. (1989) indicate that induced radioactivity contributes significantly to the low-energy region, up to about $3 \mathrm{MeV}$.

To our knowledge the features of the spatial distribution and flux composition of gamma radiation related to trapped particles in the geomagnetic field have not yet been studied in detail. For this purpose we use gamma-ray data from the low-altitude CORONAS-I experiment (Kuznetsov et al., 1991). Being a secondary product of the energetic charged particles at various pitch angles, the gamma-ray observations here are studied in the $L-B$ coordinate system, where the omnidirectional flux can be well organized. Contrary to the gamma rays the fluxes of radiation belt particles have been investigated for a relatively long time. The distribution of the fluxes of very high energy protons, electrons and positrons as a function of the magnetic adiabatic variables, have been recently reported by Fiandrini et al. (2002, 2004). In the first part of this work (Sect. 2) we concentrate on chargeparticle data interpretation in $L-B$ space at constant altitude in the Earth's magnetic field. The qualitative picture of the observed gamma-ray flux pattern in $L-B$ coordinates is discussed in the Sect. 4.

\section{Data in $L-B$ space at constant altitude}

In a dipolar approximation of the Earth's magnetic field, the physical region in $L-B$ space is bounded by magnetic field strength at the Earth's surface (or at the top of the atmosphere) and a magnetic field at the equator (i.e. minimum $B$ value) (Roederer, 1970). A level of constant altitude intersects the magnetic $L$ shell in a single $B$ value. Therefore, the fixed altitude above the Earth's surface in $L-B$ coordinates

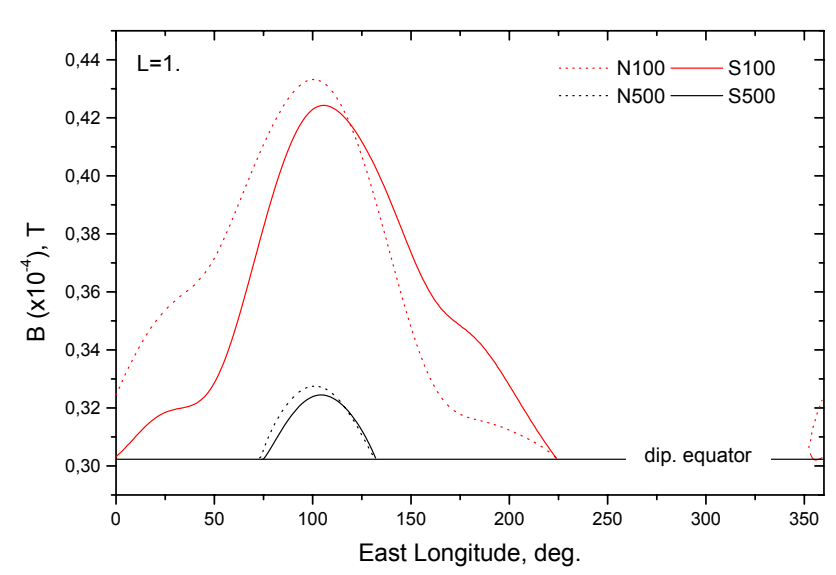

Fig. 2. The longitude spread of northern (dot) and southern (solid) magnetic mirror points, both at 100 (red) and $500 \mathrm{~km}$ (black) altitudes at magnetic shell $L=1$. The solid horizontal line indicates the magnetic equator in dipole approximation (i.e. no longitude dependent).

presents a single line starting at the magnetic equator and approaching the maximum $B$ at this altitude. However, due to azimuthal asymmetry of the real geomagnetic field, a constant altitude on different longitudes intersects the magnetic $L$ shell at various $B$ values. Thus, we have a range of $B$ values (between maximal and local minimal $B$ ) for every $L$ shell. Therefore, in $L-B$ space, the constant altitude is bounded by two lines, namely, a line of the local $B$ minima $(B \min )$ and a line of $B$ maxima (Bmax) through the magnetic $L$ shells. This is demonstrated in Fig. 1, where altitudes of $100 \mathrm{~km}$ and $500 \mathrm{~km}$ are displayed for $L>1$. The solid lines marked by Bmin 100 and Bmin500 are computed only for those $L$ shells which appear in the whole longitude range, either at $100 \mathrm{~km}$ or $500 \mathrm{~km}$ altitudes. Note that the small value $L$ shells in the non-dipolar field, close to $L=1$, do not complete the whole revolution around the solid earth. The $B$ minima for the smallest $L$ s are approximated by dipolar equatorial $B$ values (dotted line). The Bmax, Bmin lines have been obtained from a $2^{\circ} \times 1^{\circ}$ longitude-latitude grid of $L, B$ values in geodetic coordinates at altitudes of $100 \mathrm{~km}$ and $500 \mathrm{~km}$. The $L, B$ coordinates are determined by the IGRF 1990 magnetic field model. The two vertical dashed lines display $L$ shells at 1 and 1.099 . The significance of the latter one is mentioned below. Note that, due to the nondipolar nature of the Earth's magnetic field, $L$-values less than 1.0 are also possible. These $L$ shells are, however, not important for the present analysis.

From Fig. 1 it is seen that some of the diagram points-drift shells are common for both altitudes. Specifically, drift shells at $500 \mathrm{~km}$ in the area above Bmin 100 also appear at an altitude of $100 \mathrm{~km}$, often considered as a limit between zero and total particle absorption in the Earth's atmosphere (Vampola and Gorney, 1983). On the other hand, in the region below Bmin 100, drift shells never descend into the dense atmospheric layers. The charged particles on these drift shells are 


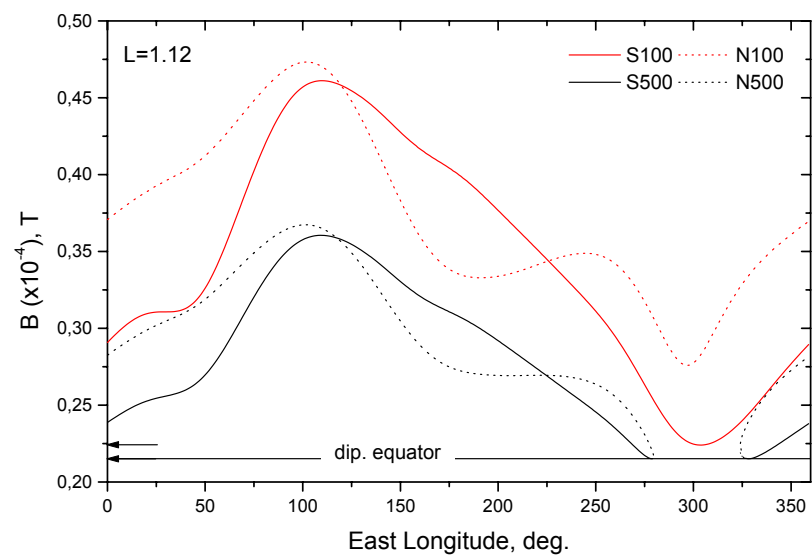

Fig. 3. Same as Fig. 2 for the magnetic shell $L=1.12$.

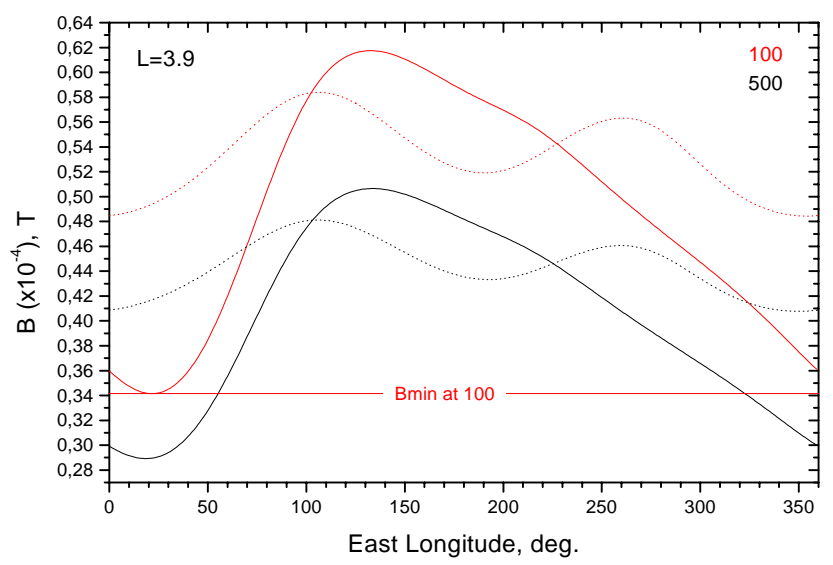

Fig. 4. Same as Fig. 2 for the magnetic shell $L=3.9$.

mostly stably trapped. One can find that this so-called stably trapped region starts at $L \sim 1.099$. In Figs. A1, A2 in Appendix A we see examples of stably trapped areas (shaded) determined by the Bmin 100 line at altitudes of 500 and $1000 \mathrm{~km}$ in geographic coordinates.

The longitude variations of magnetic field $B$ values at 100 and $500 \mathrm{~km}$ for $L$ shells equal to $1.0,1.12$ and 3.9 are illustrated in Figs. 2, 3, and 4, respectively. Figure 2 shows that at $L=1.0$ both southern and northern magnetic mirror points at an altitude of $500 \mathrm{~km}$ fall below $100 \mathrm{~km}$ altitude at some longitude. For example, the maximum $B$ value at $500 \mathrm{~km}$ - Bmax 500 (located at north) appears at $100 \mathrm{~km}$ near the $160^{\circ}$ East Longitude (EL). This is the reason why a stably trapped particle population cannot exist at this and similar small $L$ shells value.

In Fig. 3, the arrows indicate the range of $B$ values (up to Bmin 100) at magnetic shell $L=1.12$ with a minimum longitude traced altitude - Hmin above $100 \mathrm{~km}$. The charged particles having mirror points in this $B$ field interval can be stably trapped. The $L$ in Fig. 3 is an example of the magnetic shell close to 1.099. As is demonstrated in Fig. 1, below this boundary value the stably trapped population cannot be maintained.

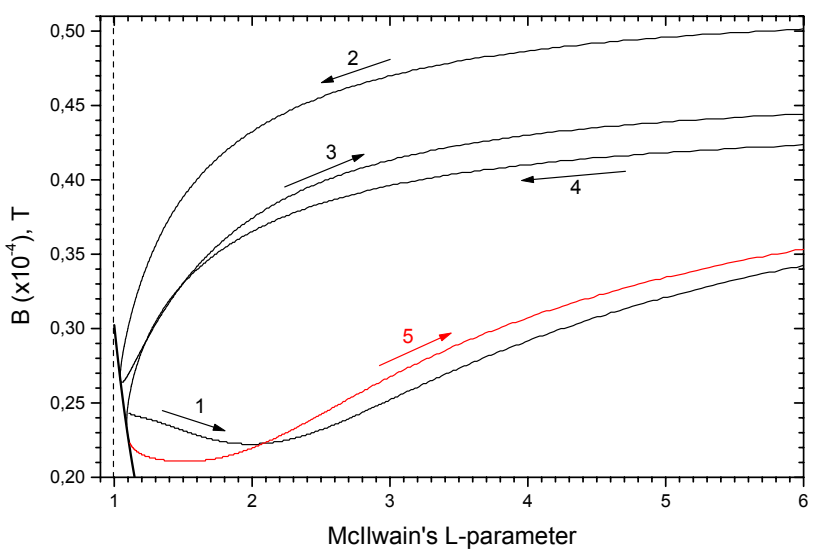

Fig. 5. The trajectory of CORONAS-I in $L-B$ space for a single orbit on day 25 June 1994 (black line). The part of the next orbit is displayed by red. The direction of motion of CORONAS-I is indicated by arrows. Thick line in the left corner indicates dipolar magnetic equator.

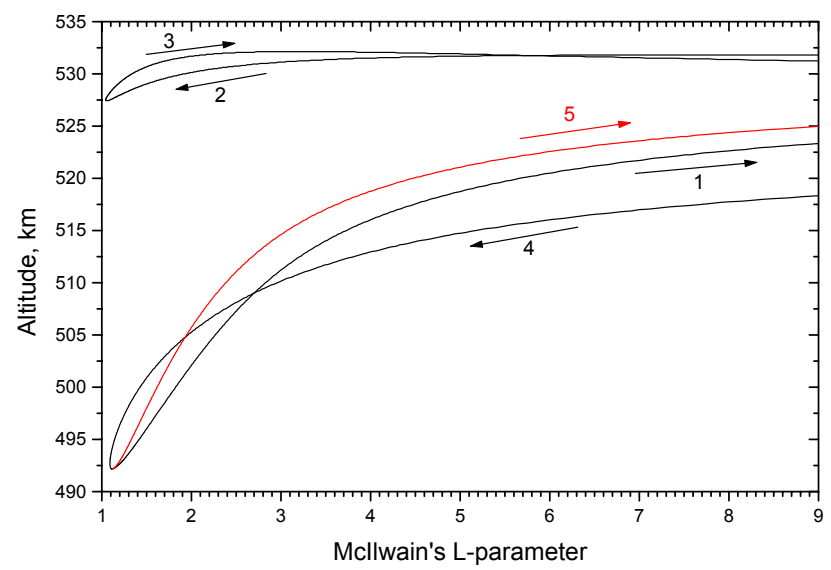

Fig. 6. The altitude of the CORONAS-I vs. $L$-shell parameter illustrates the satellite altitude variation for a single orbit on day 25 June 1994 (black line). The part of the next orbit is displayed by red. The direction of satellite motion is indicated by arrows.

The longitude distribution of mirror points at altitudes of 100 and $500 \mathrm{~km}$ for $L=3.9$ (Fig. 4) shows that in longitude intervals of $\sim 0^{\circ}-55^{\circ} \mathrm{EL}$ and $\sim 320^{\circ}-360^{\circ}$ EL the southern mirror points at $500 \mathrm{~km}$ are below $B \min 100$, and therefore lie in the stably trapped region. On the contrary, the northern mirror points at $500 \mathrm{~km}$ have in the same longitude range conjugate points below altitude $100 \mathrm{~km}$, and a bouncing motion along the field line directs particles into the atmosphere. At these longitudes, particles are in the Bounce Loss Cone (BLC) in the Northern Hemisphere. Notice that any intersection of the solid line (southern field value) with the dotted line (northern field) indicates a magnetically conjugate mirror point. In the rest of the longitude interval $\left(\sim 55^{\circ}-320^{\circ}\right)$, both northern and southern mirror points at $500 \mathrm{~km}$ have conjugate points above $100 \mathrm{~km}$ altitude. However, trapped particles in the above longitude interval, in the course of 

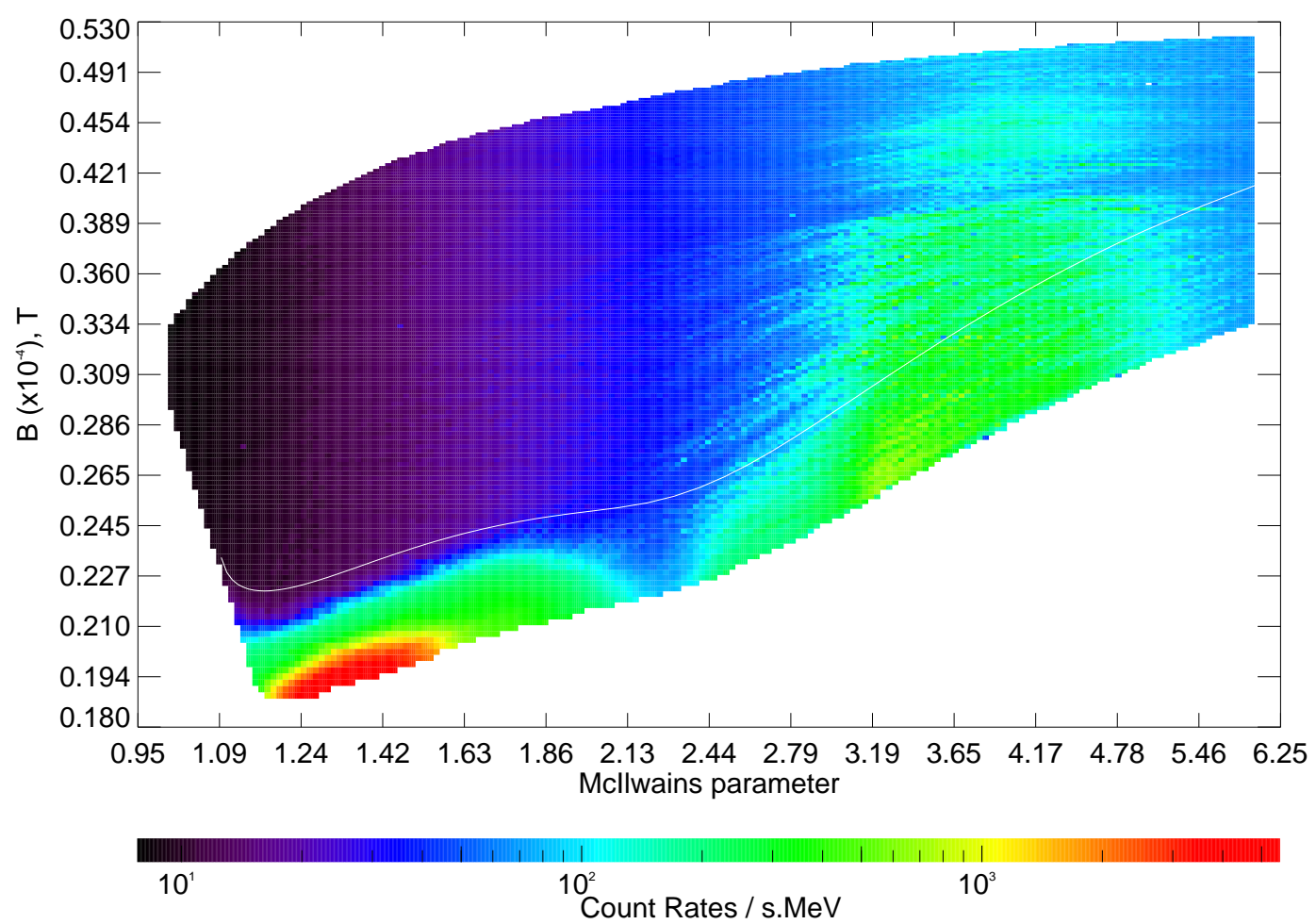

Fig. 7. The distribution of the average count rates for $3-8.3 \mathrm{MeV}$ gamma rays at CORONAS-I altitude in $L-B$ coordinates during the period March-June 1994. The Bmin 100 line is displayed by white. Colored scale matches the log of the counts.

their azimuthal drift, encounter dense atmosphere at $100 \mathrm{~km}$, forming a quasi-trapped particle population in the so-called Drift Loss Cone (DLC). The concept of the atmospheric loss cones in the geomagnetic field has been thoroughly discussed by Vampola and Kuck (1977).

\section{Experiment}

Gamma-ray data used in this study were obtained by the SONG instrument on CORONAS-I satellite. The CORONAS-I was launched on 2 March 1994 into a nearly circular orbit at an altitude of $500 \mathrm{~km}$ and an inclination of $83^{\circ}$. The Sun-synchronous orbit had a period of $\sim 95 \mathrm{~min}$. The satellite trajectory in $L-B$ space and spacecraft altitude variation for a single representative orbit, on day 25 June 1994, is demonstrated in Figs. 5 and 6, respectively. During the first working period (until 5 July 1994) the satellite was three-axis stabilized and directed with its longitudinal axis towards the Sun. The SONG device was mounted on the platform for scientific instruments at a distance of one meter from the forward end of the satellite.

The SOlar Neutron and Gamma ray (SONG) instrument consisted of a large area $\mathrm{CsI}(\mathrm{Tl})$ crystal scintillator $(20 \mathrm{~cm}$ diameter $\times 10 \mathrm{~cm}$ length) viewed by three photomultipliers. The whole scintillator counter was entirely surrounded by a 2-cm thick plastic scintillator anti-coincidence shield for charged particles. It was viewed by another three photomultiplier tubes. The CsI spectrometer had no collimator and its axis was parallel to the longitudinal axis of the satellite. Neutron and gamma rays were distinguished according to the shape of the light impulse in the CsI(Tl), which depends on the ionization of the detected particles (Dmitriev et al., 1993). The detector covers the energy range from $0.12 \mathrm{MeV}$ to $116 \mathrm{MeV}$ in nine channels. However, five energy channels between 8.3 and $116 \mathrm{MeV}$ worked episodically (data during only a couple of orbits in 1995 were recorded) and did not provide statistically significant data coverage. Here we analyse the monitoring mode of measurements with 2.5-s temporal resolution in one energy channel measuring gamma rays between 3.0 and $8.3 \mathrm{MeV}$. The instrument's effective photopeak area (at vertical incidence) is $90 \mathrm{~cm}^{2}$ at $3.2 \mathrm{MeV}$, and $61 \mathrm{~cm}^{2}$ at $7.9 \mathrm{MeV}$. More details are given in Baláž et al. (1994).

The efficiency of the active anticoincidence shield was tested by muons of the cosmic rays in the laboratory measurements. Observations by the SONG device outside the regions of trapped and quasi-trapped particles show excess count rates in the energy channels 48-68, 68-116 MeV (Ryumin et al., 1996). Authors stressed that relativistic charged particles can release energy in these energy channels where the efficiency of the anti-coincidence protection for rapid charged particles is only $\sim 95 \%$. Note that also, in the low energy channels where the efficiency of the active veto is the highest and the anti-coincidence plastic scintillator even makes a passive barrier for electrons with energies $<4 \mathrm{MeV}$ and protons with energies $<48 \mathrm{MeV}$, the positive false events 


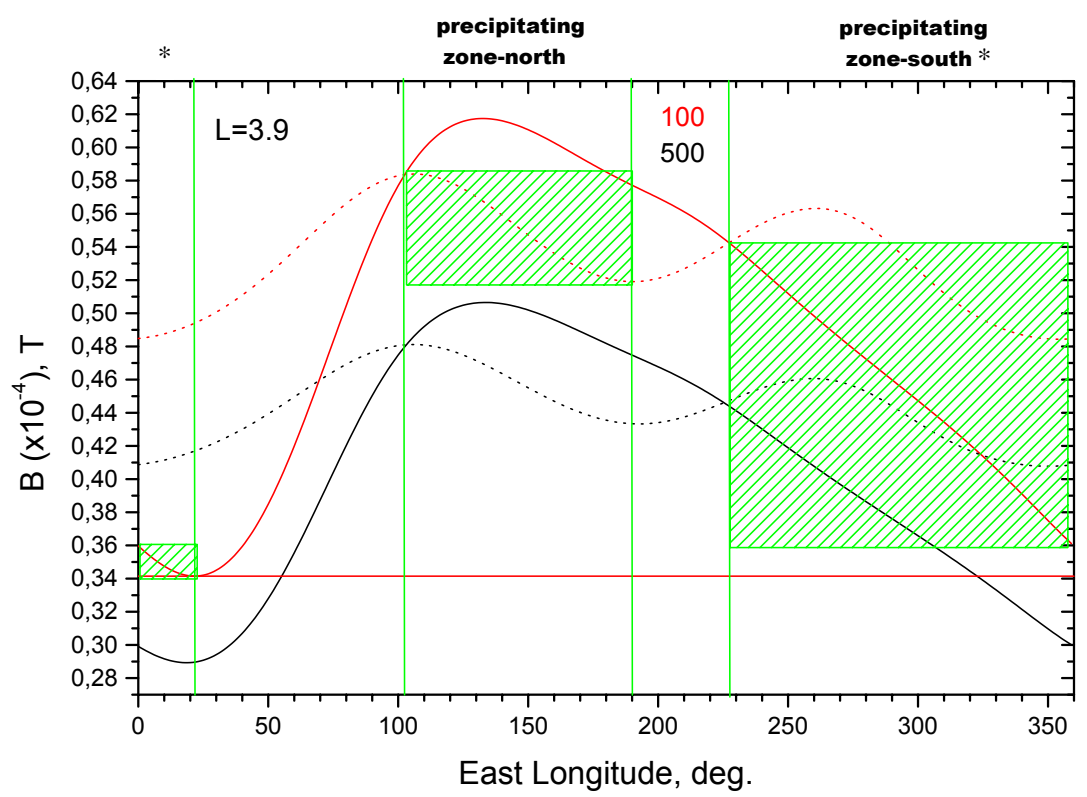

Fig. 8. The longitude intervals of the southern and northern precipitation zones at $L=3.9$ are indicated by green dashed areas. The remaining graphics have the same meaning as in Fig. 4.

in the $3-8.3 \mathrm{MeV}$ channel, due to charged particles, especially in the regions of their strong fluxes (as in radiation belts), can increase the background level.

\section{Gamma ray observations in $L-B$ space}

An overview of CORONAS-I observations of the 3-8.3 MeV gamma rays over the period from March through June 1994 in the $L-B$ coordinate system is shown in Fig. 7. The data have been divided into $180 \times 180 \mathrm{~L}-B$ bins with a step of $1.01^{i} \times 10^{-2}$ (where $i=0, \ldots, 179$ ) in $L$, and of $200 \mathrm{nT}$ in $B$. The average number of measurements in one individual bin is 50 , and the total amount of data in the selected $L$ range is $1.05 \times 10^{6}$ data points. Each $L-B$ bin is color-coded in accordance with the average gamma ray count rates.

Inspection of the $L-B$ map shows two regions of the enhanced gamma-ray emission, below $L \sim 2$ and above $L \sim 2.5$, respectively, which are separated by a region of low intensity. The observed increase in gamma-ray emissions roughly agrees with the nominal location of two zones of high intensities of energetic $(>1 \mathrm{MeV})$ electrons, and of a single (inner) zone of energetic $(>10 \mathrm{MeV})$ protons (Walt, 1994).

\subsection{Inner zone}

Figure 7 shows that enhanced gamma-ray emissions in the inner zone, at $L<2$, are well organized according to the Bmin 100 line, indicating their close connection to the stable trapped particles. It is therefore reasonable to surmise that the majority of the observed gamma rays could be produced by a stably trapped particle population in any local matter near the detector. The observations of very high energy charged particles at $400 \mathrm{~km}$ show clearly a starting point of a stably trapped region as a function of the magnetic field value (Fiandrini et al., 2004). Since the energetic trapped protons contribute mostly below $\sim 3 \mathrm{MeV}$, we suppose that the SONG gamma-ray counting rates in the channel of $3-8.3 \mathrm{MeV}$ are mainly due to local electron bremsstrahlung emission. However, further work is needed to carefully discuss the observed flux composition. An estimation of gamma-ray production by the fluxes of trapped electrons and protons of the empirical radiation models (Vette, 1991; Sawyer and Vette, 1976) in a simple evaluation of the matter distribution around the CsI on CORONAS-I (Bučík and Kudela, 2003) is in progress.

At a specific point in the $L-B$ diagram in Fig. 7, both mirroring particles and particles mirroring deeper below the satellite altitude may contribute to the gamma-ray production. This can be an explanation for the observed structure in the gamma-ray intensity distribution with emission enhancement increasing towards the equator. Since all particles mirroring along the field line pass through the magnetic equator in the course of their latitudinal bounce displacement, the flux could rise gradually as a magnetic field approaches its minimal value. However, instead of a slowly varying flux with field $B$ we measure the steep gradient of the gamma-ray count rates in the inner zone. Lemaire et al. (1995) discussed that in the inner radiation belt the steep gradient of particle flux is controlled by the density distribution of the Earth's atmosphere.

\subsection{Outer zone}

Figure 7 shows that contrary to the inner zone, the enhanced emission in the outer radiation belt, $L>2.5$, also appears outside the stably trapping. The weaker gradient of the 


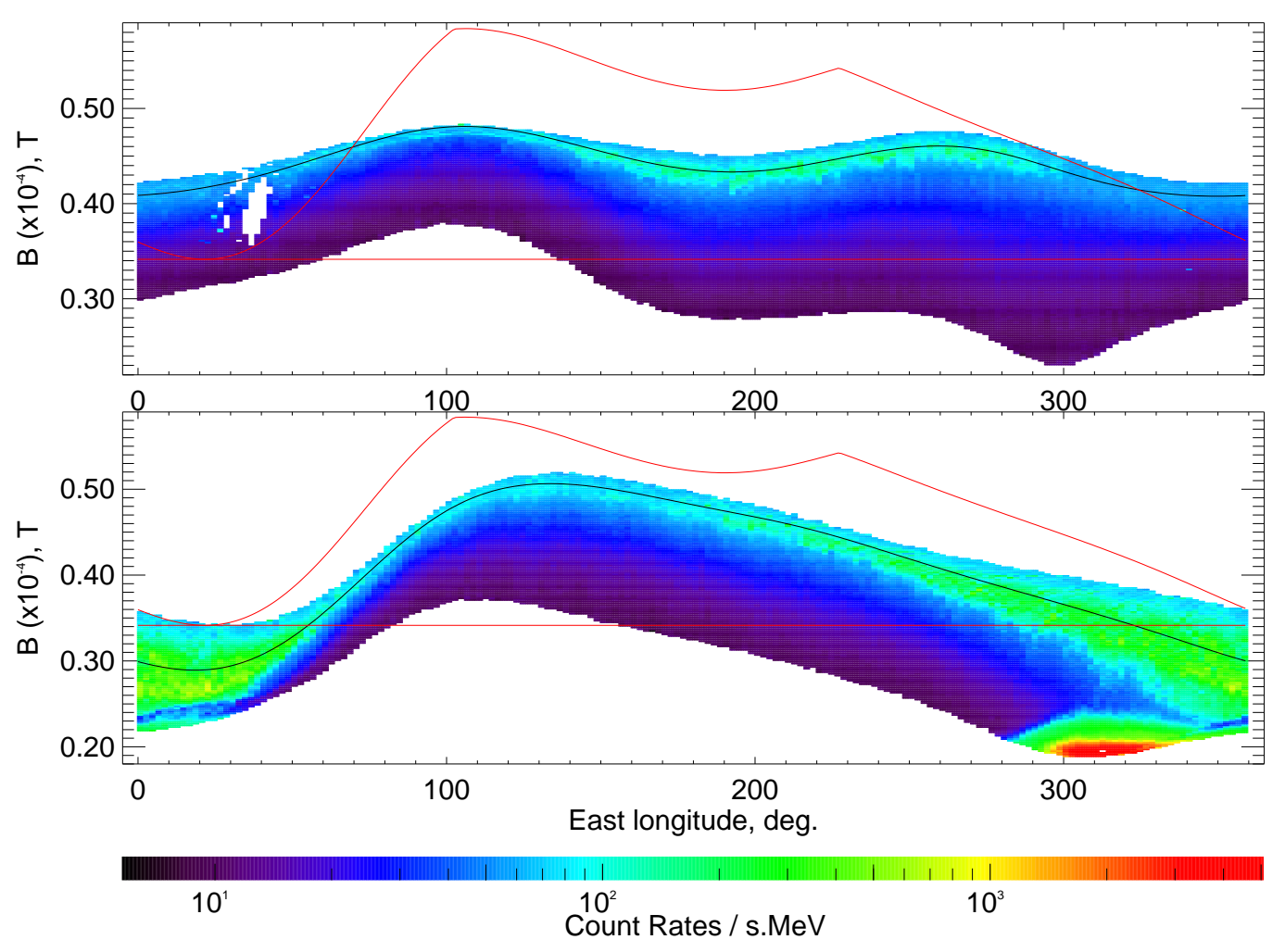

Fig. 9. The longitude distribution of 3-8.3 MeV CORONAS-I gamma rays at Northern (top) and Southern (bottom) Hemispheres for $L$-shells between 1.2 and 6 . The black line indicates $L=3.9$ at $500 \mathrm{~km}$ altitude, and atmospheric loss cones at $L=3.9$ are illustrated by the red curves.

gamma-ray count rate in the stably trapped region, observed here, is consistent with the fact that the atmospheric cutoff for the outer belt particles takes place far from the magnetic equator, where the overall flux has a maximum. Since the energetic $(>10 \mathrm{MeV})$ trapped protons are confined to the inner belt with an intensity maximum around $L \sim 1.5-2$, as indicated by the AP8 model (Sawyer and Vette, 1976), the gamma radiation in the region of the outer zone is essentially due to radiation belt electrons.

In order to expand this study to clarify measured gammaray enhancement over all sampled mirror points in the outer radiation belt, we examine the longitudinal distribution of CORONAS-I gamma-ray data. Note that the longitudinal electron distribution in the DLC is a result of competition between pitch angle scattering and azimuthal drift, and only if there is very weak diffusion, the drift takes electrons toward the eastern loss cone boundary; in the opposite case losses into the atmosphere are distributed evenly in longitude (Selesnick et al., 2003). We start first with the illustration of the longitude intervals of the southern and northern DLC precipitation zones in the outer radiation belt (at $L=3.9$ ), which is diagrammatically given in Fig. 8. In the indicated longitude intervals in the south $\left(\sim 0^{\circ}-20^{\circ}\right.$ and $\left.\sim 230^{\circ}-360^{\circ}\right)$ and in the north $\left(\sim 100^{\circ}-190^{\circ}\right)$ the quasi-trapped electron population, build up during eastward azimuthal drift, due to continuous injections over longitudes is destined to enter the altitude of $100 \mathrm{~km}$. We assume the same longitudes on the altitude of the CORONAS-I satellite, which roughly approximate regions of related DLC electrons-bremsstrahlung radiation emitted from the atmosphere. It is reasonable to expect within these longitudes a certain contribution due to locally produced bremsstrahlung from DLC electrons stopped in the satellite matter. Since the probability for production of bremsstrahlung photons is inversely proportional to the radiation length (Rossi and Olbert, 1970), and one can find that the photon production in aluminium (an element, typical in construction material) is higher by about a factor of $\sim 1.5$ than in atmosphere, this type of emission may be considerably high. Referring to Fig. 8, one can also find that the west part of the stably trapped region at an altitude of $500 \mathrm{~km}\left(\sim 320^{\circ}-360^{\circ}\right.$, and $\left.\sim 0^{\circ}-20^{\circ}\right)$ is covered by the atmospheric DLC precipitation area; therefore, the mixture of bremsstrahlung radiation both from a stable and quasitrapped electron population could be present here.

Figure 9 shows the longitude distribution of $3-8.3 \mathrm{MeV}$ CORONAS-I gamma rays at the Northern and Southern Hemispheres for $L$-shells between 1.2 and 6 over the studied time period. Each $200 \mathrm{nT} B$ (magnetic field strength) by $2^{\circ}$ longitude bin is color-coded by the average counts. The black line indicates $L=3.9$ at $500 \mathrm{~km}$ altitude. Superimposed on this distribution are loss cones at $L=3.9$, drawn by the red curves. The stably trapped zone is situated below the 


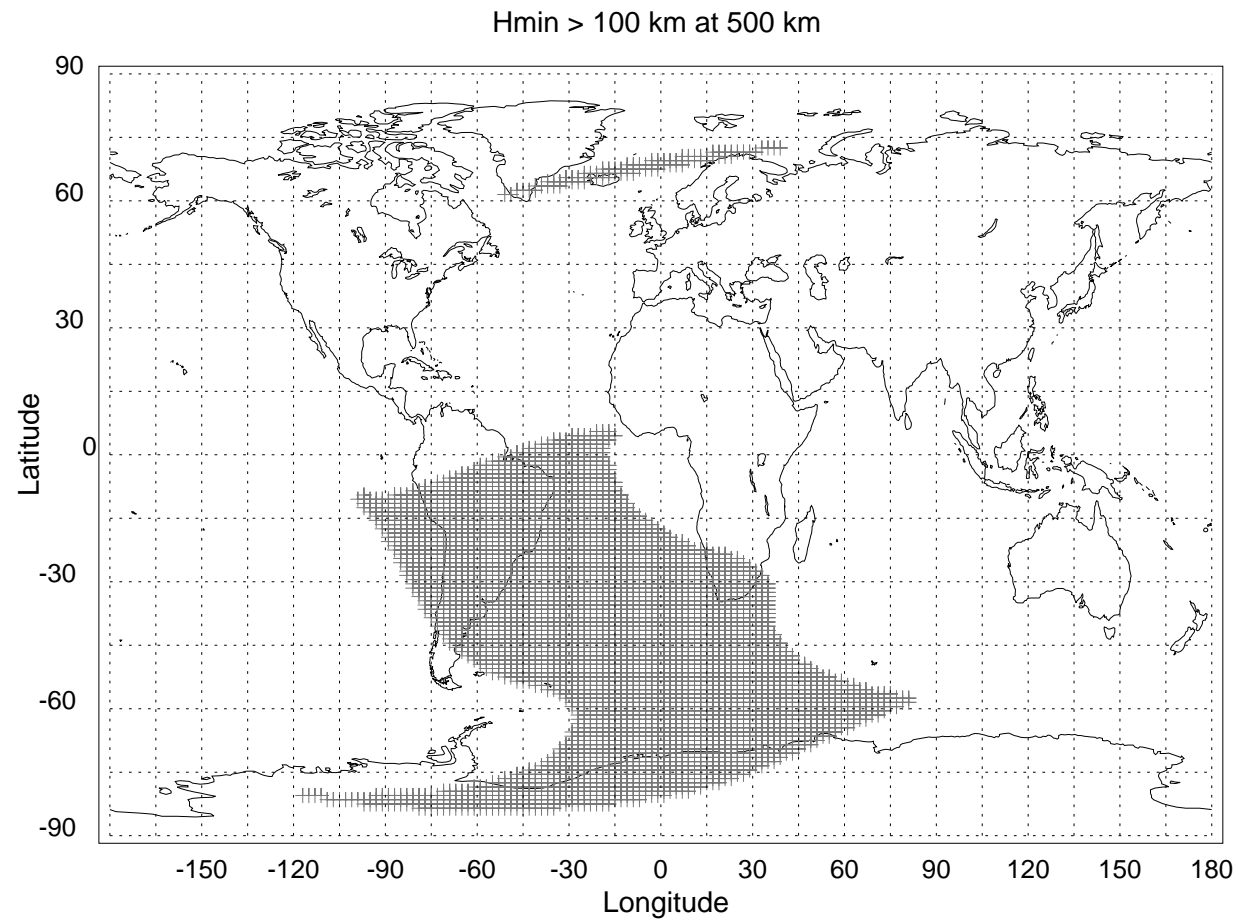

Fig. A1 The shaded areas indicate the stable trapped regions at $500 \mathrm{~km}$ for $L$ shells less than 8 .

red horizontal line (Bmin 100); above this line the DLC area is bounded by a mountain-shaped red curve. The remaining place on the graph pertains to the BLC.

From the data of Fig. 9 it is seen that the gamma-ray emission at $L=3.9$ is greater in the Southern Hemisphere, however, there are considerable longitude variations (about one order of magnitude) in both hemispheres. Some features of the variations can be understood with reference to Fig. 8 . High counting rates from the Southern Hemisphere in longitude intervals from $\sim 330^{\circ}$ to $360^{\circ}$ and from $0^{\circ}$ to $\sim 50^{\circ}$ are likely due to stably trapped electrons, although with some contribution due to directly precipitated and quasi-trapped electrons. At the Northern Hemisphere in the same longitude range, the gamma-ray intensity in the BLC is comparable with the background rate. Inspection of the variation in DLC $\left(\sim 60^{\circ}-320^{\circ}\right)$ reveals that a low count rate on the west for both north and south in some degree gradually increases with longitude. It would be consistent with gamma-ray production by DLC electrons accumulating during the eastern azimuthal drift before their precipitation. This is the clear evidence that the majority of the CORONAS-I gamma rays in the outer belt region are due to electrons characterized by eastward drift motion. The predicted positions of atmospheric DLC precipitation longitudes in Fig. 8 are not, however, in exact agreement with observed ones, in particular in the north. It is likely that gamma-ray enhancements observed in the eastern part of DLC in the north result from satellite irradiation by DLC electrons. Similarly, one can expect an enhanced local contribution during the southern satellite path to the east of DLC.
The magnetic local time dependence in electron diffusion rates, as well as transient effects of varying magnetospheric conditions, can affect the longitudinal distribution in the DLC region. Through the time of observations (MarchJune) the CORONAS-I orbit covers all local time sectors: near the dawn-dusk plane in the beginning of the period, with a consecutive orbit precession to the noon-midnight meridian one month later. Furthermore, the first half of this period was fairly geomagnetically disturbed, with three magnetic storms characterized by Dst minima falling below $100 \mathrm{nT}$. The response of CORONAS-I electron data to these storm events has been recently reviewed (Kuznetsov and Myagkova, 2002).

\section{Conclusions}

The distribution in $L-B$ coordinates based on a four-month period of CORONAS-I observations reveals that the most intense gamma-ray emissions in the $3-8.3 \mathrm{MeV}$ channel occur in the inner and outer radiation belt regions. The reconstruction of the model of $L-B$ space at constant altitude from geomagnetic field data is used to accurately interpretate the CORONAS-I measurements.

We have shown that enhanced counts in the inner belt are well confined to the region where permanently stably trapped particles are present. Then they could serve as a local source of the gamma-ray radiation when these particles hit the CORONAS-I spacecraft. In the studied energy range the electron bremsstrahlung is believed to be the most favorable production mechanism. Another contribution may 


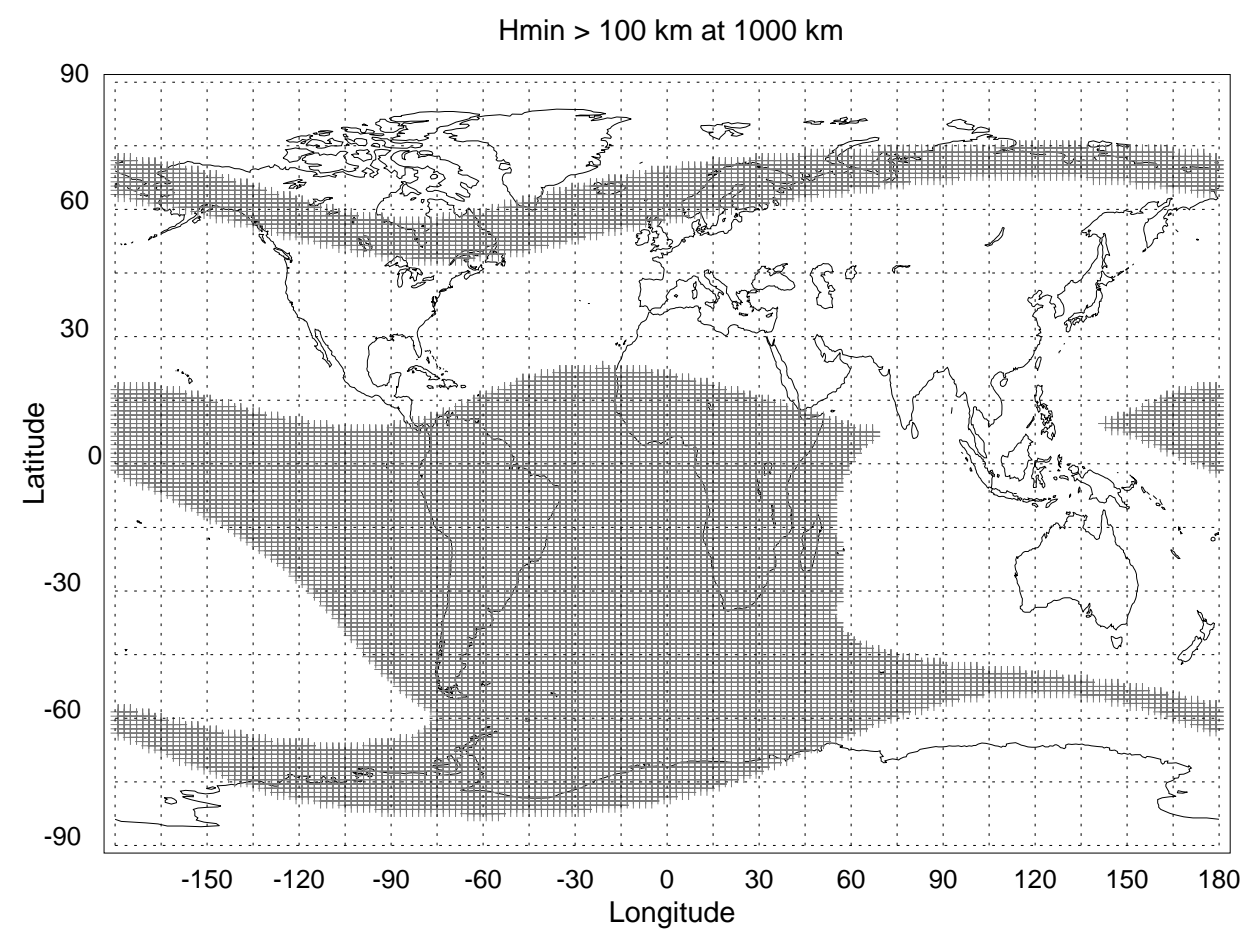

Fig. A2 Same as Fig. A1 for altitude of $1000 \mathrm{~km}$.

arise from quasi-trapped electrons near the edge of the local bounce loss cones that are lost within the atmosphere under the stable trapped region. In order to clarify this point we need to study the longitudinal variations in the inner belt region, as well.

Spacecraft observations in the outer radiation belt show that $\mathrm{MeV}$ gamma-ray enhancements have quite a complex longitude structure, different for the Northern and Southern Hemispheres. An increasing counting rate toward the eastern DLC boundary strongly suggests electrons as a source of the observed gamma radiation, both in the spacecraft matter and in the atmosphere. Further work will involve attempting to properly distinguish between local and atmospheric gammaray production.

The portion of the internal background particularly within the inner belt still remains unclear. Details about the charged particle veto system efficiency and related questions on the internal background noise in the SONG detector, especially due to high fluxes of radiation belt electrons and protons, will be discussed in the next paper.

\section{Appendix A}

Figures A1 and A2 illustrate stably trapped regions (shaded) in geographic coordinates at altitudes of 500 and $1000 \mathrm{~km}$, respectively. These zones consist of a set of $L, B$ points, for which $L$ is less than 8 and $B$ is below $B \min 100$.

Acknowledgements. This work was supported by Slovak Scientific Grant Agency VEGA project 2/4064/04. R.B. was supported by the
European Commission through the Contract No. HPRN-CT-200100314.

Topical Editor T. Pulkkinen thanks E. Finadrini and another referee for their help in evaluating this paper.

\section{References}

Baláž, J., Dmitriev, A. V., Kovalevskaya, M. A., Kudela, K., Kuznetsov, S. N., Myagkova, I. N., Nagornikh, Yu. I., Rojko, J., and Ryumin, S. P.: Solar flare energetic neutral emission measurements in the project CORONAS-I, IAU Colloq. 144, Solar Coronal Structures, edited by: Rušin, V., Heinzel, P., and Vial, J.-C., VEDA, Bratislava, 635-639, 1994.

Bučík, R. and Kudela, K.: On mass in $4 \pi$ solid angle around SONG CsI scintillator aboard CORONAS-I satellite, Acta Phys. Slovaca, 53, 329-345, 2003.

Chupp, E. L.: Gamma-ray Astronomy, D. Reidel, Dordrecht, Holland, 222-235, 1976.

Dean, A. J., Lei, F., and Knight, P. J.: Background in spaceborne low-energy $\gamma$-ray telescopes, Space Sci. Rev., 57, 109186, 1991.

Dmitriev, A. V., Kudela, K., Kuznetsov, S. N., Myagkova, I. N., Rojko, J., and Ryumin, S. P.: The SONG device for detection of the solar flare neutron and gamma radiation in CORONAS experiment (in Russian), Izv. Akad. Nauk Ser. Fiz., 57, 27-30, 1993.

Dyer, C. S., Truscott, P. R., Hammond, N. D. A., and Comber, C.: Radioactivity induced in gamma ray spectrometers, HighEnergy Radiation Background in Space, AIP Conf. Proc., vol. 186, edited by: Rester, A. C. and Trombka, J. I., American Institute of Physics, New York, 278-288, 1989. 
Efimov, Y. E., Gusev, A. A., Kudela, K., Just, L., and Pugacheva, G. I.: Spatial distribution of albedo particles on altitudes $\sim 500 \mathrm{~km}$, Czech. J. Phys. B, 35, 1371-1381, 1985.

Feldman, W. C., Symbalisty, E. M. D., and Roussel-Dupré, R. A.: Hard X-ray survey of energetic electrons from low-Earth orbit, J. Geophys. Res., 101, 5195-5209, 1996.

Fiandrini, E., Esposito, G., Bertucci, B., Alpat, B., Battiston, R., Burger, W. J., Lamanna, G., and Zuccon, P.: Leptons with $E>200 \mathrm{MeV}$ trapped in the Earth's radiation belts, J. Geophys. Res., 107, 1067, doi:10.1029/2001JA900151, 2002.

Fiandrini, E., Esposito, G., Bertucci, B., et al.: High-energy protons, electrons, and positrons trapped in Earth's radiation belts, Space Weather, 2, S09S02, doi:10.1029/2004SW000068, 2004.

Harris, M. J., Share, G. H., and Leising, M. D.: Spatial and temporal variability of the gamma radiation from Earth's atmosphere during a solar cycle, J. Geophys. Res., 108, 1435, doi:10.1029/2003JA009958, 2003.

Imhof, W. L., Nakano, G. H., Johnson, R. G., and Reagan, J. B.: Satellite observations of bremsstrahlung from widespread energetic electron precipitation events, J. Geophys. Res., 79. 565$574,1974$.

Imhof, W. L.: Review of energetic ( $>20 \mathrm{keV}$ ) bremsstrahlung Xray measurements from satellites, Space Sci. Rev., 29, 201-217, 1981.

Johnson, W. N., Kinzer, R. L., Kurfess, J. D., et al.: The oriented scintillation spectrometer experiment: Instrument description, Astrophys. J. Suppl., 86, 693-712, 1993.

Kuznetsov, S. N., Gotselyuk, Y. V., Podorolsky, A. N., Ryumin, S. P., Fisher, S., and Kudela, K.: Scientific goals and specific devices of the Solar Cosmic Rays (SCR) scientific set of instruments in the CORONAS project, CORONAS information, vol. 3, edited by: Pflug, K., Central Institute for Astrophysics, Potsdam, Germany, 1991.

Kuznetsov, S. N. and Myagkova, I. N.: Quasi-trapped electron fluxes $(>0.5 \mathrm{MeV})$ under the radiation belts: analysis of their connection with geomagnetic indices, J. Atmos. Terr. Phys., 64, 601-605, 2002.

Lemaire, J., Johnstone, A. D., Heynderickx, D., Rodgers, D. J., Szita, S., and Pierrard, V.: TREND-2, Trapped radiation environment model development, Final Report, ESTEC/Contract No. 9829/92/NL/FM, 1995.

Lorentzen, K. R., McCarthy, M. P., Parks, G. H., et al.: Precipitation of relativistic electrons by interaction with electromagnetic ion cyclotron waves, J. Geophys. Res., 105, 5381-5389, 2000.
McIlwain, C. E.: Coordinates for mapping the distribution of magnetically trapped particles, J. Geophys. Res., 66, 3681-3691, 1961.

Millan, R. M., Lin, R. P., Smith, D. M., Lorentzen, K. R., and McCarthy, M. P.: X-ray observations of $\mathrm{MeV}$ electron precipitation with a balloon-borne germanium spectrometer, Geophys. Res. Lett., 29, 2194, doi:10.1029/2002GL015922, 2002.

Roederer, J. G.: Dynamics of Geomagnetically Trapped Radiation, Springer-Verlag, Berlin, 96-101, 1970.

Rossi, B. and Olbert, S.: Introduction to the Physics of Space, McGraw-Hill, New York, 186-191, 1970.

Ryumin, S. P., Bogomolov, A. V., Dmitriev A. V., and Myagkova, I. N.: Background conditions for recording $\gamma$ radiation and energetic neutrons on CORONAS satellites from SONG data, J. Moscow Phys. Soc., 6, 423-430, 1996.

Sawyer, D. M. and Vette, J. I.: AP-8 trapped proton environment for solar maximum and solar minimum, NSSDC 76-06, NASA Goddard Space Flight Center, Greenbelt, Md., 1976.

Selesnick, R. S., Blake, J. B., and Mewaldt, R. A.: Atmospheric losses of radiation belt electrons, J. Geophys. Res., 108, 1468, doi:10.1029/2003JA010160, 2003.

Share, G. H., Kinzer, R. L., Strickman, M. S., Letaw, J. R., Chupp, E. L., Forrest, D. J., and Rieger, E.: Instrumental and atmospheric background lines observed by the SMM gamma-ray spectrometer, High-Energy Radiation Background in Space, AIP Conf. Proc., vol. 186, edited by: Rester, A. C. and Trombka, J. I., American Institute of Physics, New York, 266-277, 1989.

Share, G. H., Murphy, R. J., Dennis, B. R., et al.: RHESSI observation of atmospheric gamma rays from impact of solar energetic particles on 21 April 2002, Solar Phys., 210, 357-372, 2002.

Vampola, A. L. and Gorney, D. J.: Electron energy deposit in the middle atmosphere, J. Geophys. Res., 88, 6267-6274, 1983.

Vampola, A. L. and Kuck, G. A.: Induced precipitation of inner zone electrons, Volume I: Observations, SAMSO-TR-77-134, The Aerospace Corporation, Los Angels, CA, 1977.

Vette, J. I.: The AE-8 trapped electron model environment, NSSDC 91-24, NASA Goddard Space Flight Center, Greenbelt, Md., 1991.

Walt, M.: Introduction to Geomagnetically Trapped Radiation, University Press, Cambridge, Great Britain, 59-86, 1994. 